\title{
Evolutionary Algorithms for QoE
}

\author{
Seyed Mahmood Hashemi
}

\begin{abstract}
Quality of Experience (QoE) is an important parameter in communication ways. The importance of $\mathrm{QoE}$ becomes more when we use Long Term Environment (LTE) to transmit data packets. However, there are some methods to measure QoE, the complexity of common methods is obstacle to use popularity. In this paper, we present an approach to measure QoE in LTE. Presented approach is usability as precise. We use three optimization algorithms in our approach: 1-intlligent automata 2-simulated annealing 3-monarchy butterfly optimization. Although the original release of used algorithms are single-objective, we improve them to multiobjective. We compare the performance of algorithms.
\end{abstract}

Index Terms - Quality of experience, long term environment, multi-objective optimization.

\section{INTRODUCTION}

In 1876, when Alexander Graham Bell invented the telephone, the communications revolution started, which has since changed the world. Over the years, massive quantitative measures have been presented to determine the desired levels of delivered quality, including Mean Time between failures, Mean Time to Repair, Delay, Jitter, Percentage of Packets Lost, Hold Time, Five Nines of Reliability, Carrier-Grade Service, Toll-Quality Voice and so on. However much these measures are useful, they are exclusively inwardlooking. In other words, they examine network performance from the server viewpoint, but the other side of this equation (performance) is declared in the user viewpoint. Thus, most service providers are now shifting their focus from quality-of-service (QoS) to qualityof-experience (QoE). Actually, watching video streams with users is one of the most important duties of LTE services. Therefore, parameters of QoE - which are important for end users to receive video streams with good qualityacquire greater importance. Any variability in communication channel characters, which affect QoE, can be analysed with statistics. Unfortunately, high computational complexity and poor flexibility are problems faced in estimating QoE parameters. Indeed, the structure of the network is suddenly changed, so that QoE cannot be strictly estimated. The aim of this paper is to present an approach to predict QoE with stochastic parameters. The major goal of this paper is ensuring quality for video streams, but it can be used for other applications without loss of generality. We present QoE as an optimization problem, so that it is more comprehensible for both users and administrators. The proposed approach uses Evolutionary Algorithms for optimization, so that it can be easily adapted to network circumstances.

Manuscript received March 12, 2018; revised May 10, 2018.

Seyed Mahmood Hashemi is with Beijing University of Technology, China (e-mail: hashemi2138@yahoo.com).
There are three algorithms based on QoE to ensure high quality video stream in LTE: 1- Packet Scheduling (PS), 2Call Admission Control (CAC) and 3- Handover Algorithm (HA). Briefly, PS decides which data packet will be transmitted in the next interval. CAC determines which user request must be serviced. HA is responsible for ensuring that the User Equipment (UE) is freely transferred. We focus on CAC, because we think it is more challengeable than the others (PS and HA). Of course, our proposed scheme is an extension of CAC and adds a chosen suitable path to it. This paper is organized as follows: Section 2 discusses QoE versus QoS. Section 3 introduces LTE. Section 4 explains the problem. In Section 5, some Related Works are investigated. Section 6 introduces Number Optimization algorithms. Section 7 is about Experimental Results and Section 8 is the Conclusion.

\section{QUALITY OF EXPERIENCE (QOE) VS. QUALITY OF SERVICE (QOS)}

Central to any discussion on internet content delivery is quality and has two common terms: Quality of Service (QoS) and Quality of Experience (QoE, or sometimes referred to as QX). These terms differ in important ways. QoS considers different parameters such as bandwidth, latency, jitter, packet loss and packet delay. However, QoS is sublimely easy to gauge (because it is a numerical measure of some aspect of network performance). QoE, in contrast, is an entirely different beast. QoE is an outward-looking measure. It means QoE is a customer-focused measure, rather than just network-oriented same as QoS. While increasing video stream request, customers care about the inner workings of network performance, particularly in light of the much more media-rich services that service providers are being asked to transport.

Thus QoE assessment is harder than QoS assessment, because QoE is subjective and QoS is objective. For a given application, you cannot be told what your QoE is or should be. It is personal, based on your experience and expectations. It can mean the difference between service abandonment and upsell. For example, the most obvious measure we have is the 'subscriber churn rate': if customers fail to receive the level of service they expect or if they fail to have a memorable experience when engaging with the network, they will vote with their wallets and subscribe to a different carrier(s). Thus, the major goal must be the satisfaction of customer expectations. This is the challenge that lies before the modern telecom service provider, as today, network traffic is growing at a precipitous rate and is becoming increasingly media-intensive, thanks to the popularity of video services. As a consequence, the revenue-pertransported bit is in sharp decline, which means that service providers must find other ways to make up the difference if they wish to remain profitable. QoE is an end-to-end 
function of the path from the user to his or her data and applications. This requires deep visibility, and some way to influence performance. Given the internet-routing limitations and no true end-to-end QoS model, we require an overlay - a way to bypass best-effort links and congestion. Therefore, delivering scalable QoE requires an overlay.

\section{LONG TERM EVOLUTION (LTE)}

On metropolitan area IEEE802.16 standard is defined (wireless network). Its original goal is to support fixed/nomadic users. It is upgraded to IEEE802.20 to support high mobility application, but its survival is in doubt. The start for standardization of Long Term Evolution (LTE) is late 2004. The first release of the LTE specifications-Release 8 -was completed in 2008. LTE is continuously being developed to ensure that future requirements and scenarios are being met and prepared for in the best manner. There are three important advantages of LTE: Performance and capacity: One of the requirements of LTE is that it should provide downlink peak rates of at least $100 \mathrm{Mbps}$. In the first stage, the technology allows for speeds of over 300Mbps. Furthermore, Radio Access Network (RAN) round-trip times are expected to be less than $10 \mathrm{~ms}$. Simplicity: LTE supports flexible carrier bandwidths from $1.4 \mathrm{MHz}$ up to $20 \mathrm{MHz}$. LTE also supports both Frequency Division Duplex (FDD) and Time Division Duplex (TDD). So far, a large number of bands has been identified by 3th Generation Partnership Project (3GPP) for LTE, and there are more bands to come. This means that an operator may introduce LTE in 'new' bands where it is easiest to deploy $10 \mathrm{MHz}$ or $20 \mathrm{MHz}$ carriers. Features like selfconfiguration and self-optimization will simplify and reduce the cost of network rollout and management. Wide range of terminals: In addition to mobile feature phones, smartphones, computers and consumer electronic devices, such as laptops, notebooks and tablets, incorporate LTE embedded modules. In the near future, other devices—-such as gaming devices and cameras-will also incorporate LTEembedded modules. Since LTE supports handover and roaming to existing mobile networks, all these devices can have ubiquitous mobile broadband coverage from Day One. In summary, operators can introduce LTE flexibly to match their existing network, spectrum and business objectives for mobile broadband and multimedia services. LTE uses Orthogonal Frequency Division Multiplexing (OFDM) as its radio access technology. OFDM meets the LTE requirement for spectrum flexibility and enables cost-efficient solutions for very wide carriers with high peak rates. It is a wellestablished technology: for example, in standards such as IEEE $802.11 \mathrm{a} / \mathrm{b} / \mathrm{g}, 802.16$, HIPERLAN-2, DVB and DAB. In the uplink, LTE uses a pre-coded version of OFDM called Single Carrier Frequency Division Multiple Access (SCFDMA). This is to compensate for a drawback with normal OFDM, which has a very high Peak to Average Power Ratio (PAPR). High PAPR requires expensive and inefficient power amplifiers with high linearity requirements, which increases the cost of the terminal and drains the battery faster. Advanced antenna solutions that have been introduced in
High Speed Packet Access (HSPA) Evolution are also used by LTE. Solutions incorporating multiple antennas meet nextgeneration mobile broadband network requirements for high peak data rates, extended coverage and extensive capacity. In addition to LTE, 3GPP is also defining IP-based, flat core network architecture. This architecture is defined as part of the System Architecture Evolution (SAE) effort specifying the Evolved Packet Core (EPC) network. The Evolved Packet System (EPS) includes EPC, Evolved Universal Mobile Telecommunication System (UMTS) and Terrestrial Radio Access Network (E-UTRAN). An EPS bearer is typically associated with a QoS. Multiple bearers can be established for a user in order to provide different QoS streams or connectivity to different Packet Data Networks (PDNs). Basically, we can say there are two types for bearers: 1Guaranteed Bit Rate (GBR) and 2- Non-Guaranteed Bit Rate (NGBR). In GBR, congestion of data packets is assumed permanently. In NGBR, congestion of data packets may be losses. The network must also provide sufficient security and privacy for the user and protection for the network against fraudulent use.

Therefore, we can find LTE elements in two groups: The Evolved Universal Terrestrial Radio Access Network (EUTRAN): LTE base station (eNodeB) Evolved Packet Core (EPC): Mobility Management Entity (MME), Policy and Sharing Rules Function (PSRF), Serving Gateways, Public Data Network (PDN) Gateway, Home Subscriber Server (HSS) In a typical case, multiple applications may be running in a User Equipment (UE) at any time, each one having different quality of service requirements. For example, a UE can be engaged in a Voice on IP (VoIP) call while at the same time browsing a web page or downloading an FTP file. VoIP has more stringent requirements for QoS in terms of delay and delay jitter than web browsing and FTP, while the latter requires a much lower packet loss rate.

In order to support multiple QoS requirements, different bearers are set up within the Evolved Packet System, with each being associated with a QoS. Thus, the gateway has to direct Data Packets on connections, which have suitable QoS.

\section{PROBLEM}

Suppose there is a LTE network with 5 eNodeBs. On one side of the network, there is a user. Each EnodeB can receive a request of video streams and send it to the server. On the other side of the network, there is a server that receives the request of the video stream from eNodeB, then sends the video stream. Finally, eNodeB sends a video stream to the user. Thus, all eNodeBs have connections with the user and also the server. This scheme is depicted in Fig. 1. Of course, the number of users, eNodeBs and servers can rise. There may be multiple servers with different services. But none of them contradicts our assumptions. Actually, our scheme is abstract, and many LTE details are omitted in it, but details can be added freely. Note that important entities for the problem are showed in our plan. 


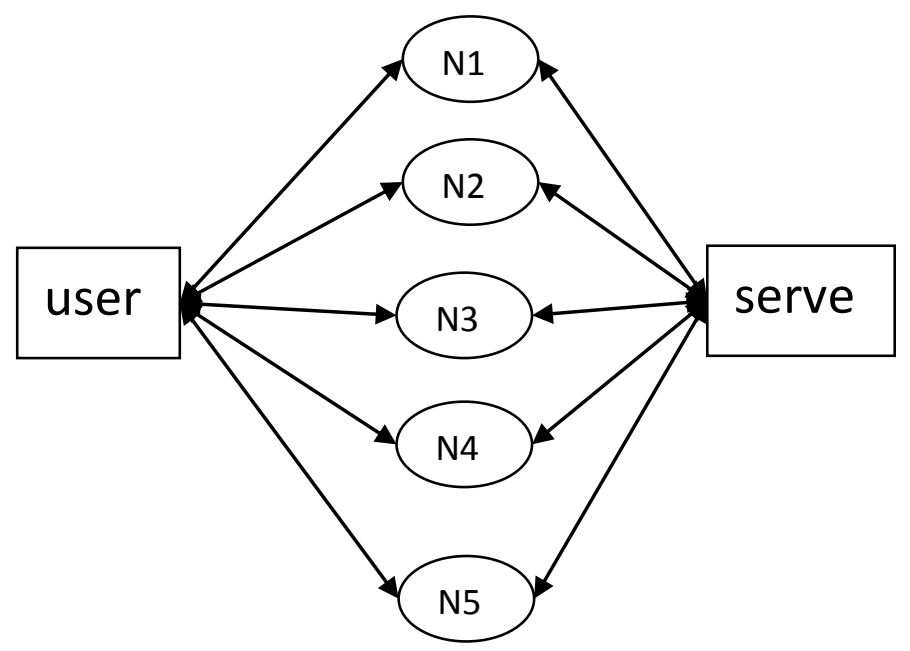

Fig. 1. Overview scheme of the network.

Our problem is choosing a suitable path to transfer the video stream while satisfying QoE. Our approach considers two parameters: 1- availability and 2- cost. Although there are many effective parameters for QoE, the basics parameters are these (availability and cost). Availability and cost are in contrast to each other, because the high degree of availability is attractive to users and also users want to pay less for their requested services. If we present and solve QoE on LTE with just these parameters (availability and cost), we have the potential to present and solve QoE with other parameters.

Video stream is encoded eNodeB before it is transmitted, so AVAILABILITY is defined as follows:

$$
C^{i}(t)=\left\{\begin{array}{lr}
A \int_{0}^{t} R_{t r}^{i}(\tau) d \tau-A *\left(t-t_{0}-T_{\text {int }}\right) ; t & \geq t_{0}+T_{\text {int }} \\
A \int_{0}^{\mathrm{t}} R_{t r}^{i}(\tau) d \tau ; & \text { otherwise }
\end{array}\right.
$$

$C^{i}$ : availability in ith eNodeB

$A$ : number of frames per second in video streaming service

$R_{t r}^{i}:$ for transmission data in ith eNodeB

$t_{0}$ : start time for requesting video stream service

$T_{\text {int }}$ : initial latency for video stream

We defined availability as time-varying, so it is denoted with $C^{i}(t)$. COST is defined by:

$$
E^{i}=A \cdot R_{t r}^{i} . D C^{i}
$$

$D C^{i}$ : default cost for ith eNodeB

Our problem is a decision-making base on QoE of each eNodeB. In other words, we must select eNodeB with high availability and low cost. We can then define our problem as an optimization problem:

$$
\begin{aligned}
& \text { Opt. QoE(i) } \\
& \text { S.T.: } i \in\{1, \cdots, N\}
\end{aligned}
$$

$$
\mathrm{QoE}(i) \equiv F\left(E^{i}, C^{i}(t)\right)
$$

The first constraint says that the QoE selected must be in a predefined set. The second constraint says QoE is defined as a function of availability (1) and cost (2).

\section{RELATED WORKS}

A major evolution in $4 \mathrm{G}$ resides in the air interface which has introduced a simplified All-IP network architecture.' Though $4 \mathrm{G}$ has many advantages, the impact of services and security on it has become critical. Typical threats in 4G include different attacks like Denial of Service (DoS) attacks. Mavoungou et al. provide an overview of threats and attacks in mobile networks. [1] In the Third Generation Partnership Project (3GPP), there are 5 features for security: Access Control: provides secure user access to the services. Domain: provides secure signalling between nodes. User Domain: provides secure access for users to the Mobile Station (MS). Application Domain: provides secure communication between entities. Visibility and configurability: Presents a degree of security to the user to inform him/herself about security. Any weakness that can be exploited by a threat can be known as a vulnerability. Various types of malware attacks threaten mobile network vulnerabilities across a broad range, including mobile network services, internet access and Bluetooth. Malware attacks can also remotely control a server. Mobile network attacks can be categorized into four groups: Loss of availability: Interface flooding and network elements crashing. In the physical layer, it can be achieved via bustling communicate path. Loss of confidentiality: Traffic eavesdropping, unauthorized data access. It can be performed with analyses of encrypted traffic or eavesdropping. Loss of integrity: Traffic modification, data modification. It is achieved with an intruder: an attacker between the target user and a genuine network to data modification. Loss of control: Compromised network element, malicious insider. Its aim is to gain control over a network element with protocol/application implementation flaw. Authentication procedures occur at every Attack Area Update (TAU), etc. In handover key management cases, a number of vulnerabilities communicate in an unsecure path. The aim of Long Term Evolution (LTE) is the provision of high data rate $(100-300 \mathrm{Mb} / \mathrm{s})$. Bagubali et al. try to integrate LTE with Wimax.[2] Equations for delay: $\square \square(\square h \square)=\square \square(\square \square \square)+\square \square(\square \square \square)+\square \square(h \square \square)$. The aim of [3] is to design a Call Admission Control (CAC) scheme based on user requirements QoS. The author supposes that the users have uniform distribution and also strictly divides the users into two groups: golden and silver. CAC is used to guarantee the QoS. It controls the number of LTE users. The architecture of LTE also called Evolved Packet System (EPS). Users can enter the network after 
their authentication. Authors of [4] propose double authentication scheme to address de-synchronization attacks. The aim of the paper [5] is to design handoff (signal transition) between different ranges. Authors present some formulas for reward and penalty functions. However, as their problem is different from our problem, the formulas presented can be useful. The major goal of the paper [6] is the optimized usage of resources in the LTE network. Authors propose using Genetic Algorithm (GA) with Random Neural Network (RNN) to optimize parameters of QoE. Gago et al. analyse the LTE network capacity by considering both Inter Cell Interference Coordination (ICIC) and Economics to reach a conclusion.[7] Authors use Vienna System Level Simulator to test fomtocell over LTE. Abdeljebbar et al. proposed a mechanism for handover in LTE network, such that they explain the security system in LTE as a hierarchical key system.[8] They try to reduce the latency for authentication while using a Handover Keying Working Group (HOKEY). Their definition of LTE components is useful. Deng et al. propose an algorithm to estimate the channel for

LTE-railway.[9] They use Basis Expansion Model (BEM) to estimate dynamic change with Time-Varying (TV). However much their formulas are attractive, they are not useful for us while undertaking difference architecture. Wan et al. propose a cross-layer approach with Packet Scheduling (PS), Call Admission Control (CAC) and Handover Algorithm (HA).[10] For PS, they propose to regulate video data to avoid buffer underflow. For CAC, they use Poisson's distribution to determine whether or not a new call request is admitted. For HA, eNodeB keeps balance of QoE states among adjacent eNodeBs. However, this paper is very limited (for selection Poisson's distribution and number of video requests), though the formulas they present are useful. In this paper, we solve (3) in accordance with (1) and (2) with numbers MultiObjective Optimization (MOO) algorithms.

\section{OPTIMIZATION ALGORITHMS}

We can divide optimization algorithms into two main groups: 1- deterministic and 2- evolutionary. However much deterministic algorithms produce optimum results, evolutionary algorithms are more useful in the network. The major constraint in the network (especially LTE) is time. Each router (eNodeB) receives and sends data packets in the hard limited time, so that they cannot wait until the optimization process in the deterministic algorithms are finished. Unfortunately, deterministic algorithms produce final results just when they are finished, so that they are not useful for routers (eNodeBs). Instead of deterministic algorithms, we can use evolutionary algorithms. Though the final result of evolutionary algorithms is not very good, they can produce the final result at any point of time, so that we can cover time limitation. Where there are multiple goals for optimization, we should use Multi-Objective Optimization (MOO). MOO is necessary when multiple cost functions are considered in the same problem. The aim of MOO is to tune the decision variables to satisfy all objective functions with optimum value. This class of problems is modelled by (1).

Optimize $[F 1(X), \cdots, F k(X)]$

$$
\text { Subject to: } g 1(X), \cdots, g m(X) \leq 0 ; h 1(X), \cdots, h p(X)=0
$$

where $k$ is the number of objective functions, $X$ is the decision vector, $\mathrm{m}$ is the number of inequality constraints, and $p$ is the number of equality constraints. This goal causes a difference between these algorithms and their ancestorSingle-Objective Optimization - which is based on the concept of the best solution, while MOO uses the concept of dominate solution. Dominance is defined in [11]:

$$
\begin{array}{r}
\vec{U}=\left(u_{1}, \cdots, u_{n}\right) \prec \vec{V}=\left(v_{1}, \cdots, v_{n}\right) \text { iff } \forall i \in \\
\{1, \cdots, n\}: u_{i} \leq v_{i}, \exists j \in\{1, \cdots, n\}: u_{j}<v_{j}
\end{array}
$$

In words, a vector $\vec{U}=\left(u_{1}, \cdots, u_{n}\right)$ dominates another vector $\vec{V}=\left(v_{1}, \cdots, v_{n}\right)$ if and only if $\vec{U}$ can reach the optimal value for some criterion without causing a simultaneous non-optimal value for at least one criterion. If two vectors cannot dominate each other, they are called non-dominated vectors. [12] In this paper, we use three Evolutionary MultiObjective Optimization algorithms: 1Multi-Objective Monarch Butterfly Optimization (MOMBO), 2- MultiObjective Intelligent Automata (MOIA) and 3-MultiObjective Simulated Annealing (MOSA).

\section{A. Monarch Butterfly Optimization (MOMBO)}

The basis of Monarch Butterfly Optimization (MBO) is to move the characteristics of a monarch butterfly [13]-[15]. The same as other swarm optimization algorithms, there is a set of individuals. Individuals are evaluated in accordance with objective functions. Since there are multiple objective functions, we must use a dominance concept, but in $\mathrm{MBO}$, there is a different scenario. In MBO, butterflies (individuals) in different lands are updated with migration operator. So there is no need to undertake a comparison with dominance. MBO (Fig. 4) includes two algorithms: 1Migration and 2- Adjusting.

\section{B. Multi-Objective Intelligent Automata (MOIA)}

Automata can be represented by five tulles $\mathrm{SA}=$ $\{\alpha, \beta, F, G, \Theta\}$, where $\alpha$ is a set of actions, $\beta$ is a set of inputs of automata, $\mathrm{F}$ is a function that maps the input and current state to the next state $F \equiv \Theta \times \beta \rightarrow \Theta, G$ is the output function that maps the current state to the next output, and $\Theta$ is a set of states. An automaton selects an action in $\alpha$ at each iteration. If the mapping of $\mathrm{F}$ and $\mathrm{G}$ is deterministic, then the automata are called deterministic; otherwise, the automata are called stochastic. The output and next state of deterministic automata are achieved uniquely in accordance with the initial state and inputs. For stochastic automata, we can only determine the probability of the output and next state. Stochastic automata are divided into two categories: fixed structure and variable structure. In fixed-structure stochastic automata, the probabilities of the next states and outputs are constant at each iteration; however, in variablestructure stochastic automata, they are changed.

We can divide learning algorithms into two categories: standard algorithms and model algorithms. In standard algorithms, the formula for learning is $\mathrm{P}(\mathrm{n}+1)=T[\mathrm{P}(\mathrm{n}), \alpha$ $(n), \beta(n)]$, where $P$ is the probability of selection of an action and $\mathrm{T}$ is a learning algorithm, which may be linear or non-linear. In other words, learning algorithms increase the probability of selection of action $\alpha \mathrm{i}$ and decrease the 
probability of selection of other actions, if the desired response is received from the environment for action $\alpha \mathrm{i}$ at the nth iteration. Otherwise, they decrease the probability of selection of $\alpha \mathrm{i}$ and increase the probability of selection of other actions. Therefore, we can say that the probability of its selection is increased when the new state dominates the previous state.

$$
P(n+1)=P(n)+a P(n)
$$

Again, the probability is decreased when the new state is less than the previous state.

$$
P(n+1)=P(n)+b\left(\frac{1}{r-1}-P(n)\right)
$$

$a, b$ are factors for Reward and Penalty. In the approach presented, we set $\mathrm{a}=\mathrm{b}$ In the third state, a solution is nondominated with another solution(s). The selection probability in the third state does not change.

\section{Multi-Objective Simulated Annealing (MOSA)}

In Multi-Objective Simulated Annealing (MOSA), there is one individual that can be changed during process. The present individual is changed randomly and a new individual is created. Thus, we can consider two states: 1$c p$ (current point) and 2- $n p$ (new point). Both of them are randomly initialized, and then we compare them. The result of the comparison is one of these states: $c p$ dominates $n p: c p$ is not changed and $n p$ is discarded. $c p$ is dominated by $n p$ : $c p$ is replaced with $n p$ randomly. $c p, n p$ are non-dominated: $n p$ is saved in archive. When a new individual is added to the archive, it (new individual) is compared with all archived individuals.

\section{EXPERIMENTAL RESULTS}

Lets 24 frame is needed for a video stream. Default costs, ration transmission, need time for all eNodeBs are as the

\begin{tabular}{|c|c|c|c|c|c|}
\hline & $\begin{array}{l}\text { eNod } \\
\text { e B1 }\end{array}$ & $\begin{array}{l}\text { eNod } \\
\text { e B2 }\end{array}$ & $\begin{array}{l}\text { eNod } \\
\text { e B3 }\end{array}$ & $\begin{array}{l}\text { eNod } \\
\text { e B4 }\end{array}$ & $\begin{array}{l}\text { eNod } \\
\text { e B5 }\end{array}$ \\
\hline $\begin{array}{l}\text { Default } \\
\text { Cost }\end{array}$ & 0.4 & 0.5 & 0.3 & 0.7 & 0.8 \\
\hline $\begin{array}{l}\text { Transmissi } \\
\text { on Data }\end{array}$ & 0.2 & 0.2 & 0.4 & 0.6 & 0.4 \\
\hline $\begin{array}{l}\text { Needed } \\
\text { Time }\end{array}$ & 0.2 & 0.4 & 0.1 & 0.3 & 0.6 \\
\hline
\end{tabular}
following table:

Values in Table I are in $[0,1]$, but it is for normalization. Therefor, there are two variables for QoE in (3): 1availability and 2- cost. Of course, the ideal is simultaneously maximum availability and minimum cost. We use three algorithms that are explained in the previous section.
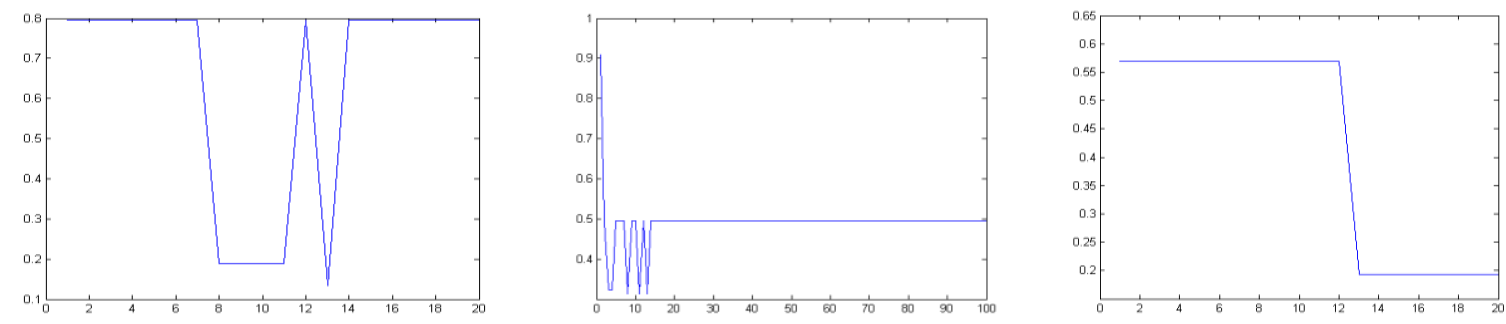

Fig. 2. Results for availability a) MOIA b) MOMBO c) MOSA (from left to right).

As you can see, the result of the availability in MOMBO is better than the results for cost in other algorithms (MOIA, MOSA). The results of MOMBO converge sooner than results of MOSA and MOIA.
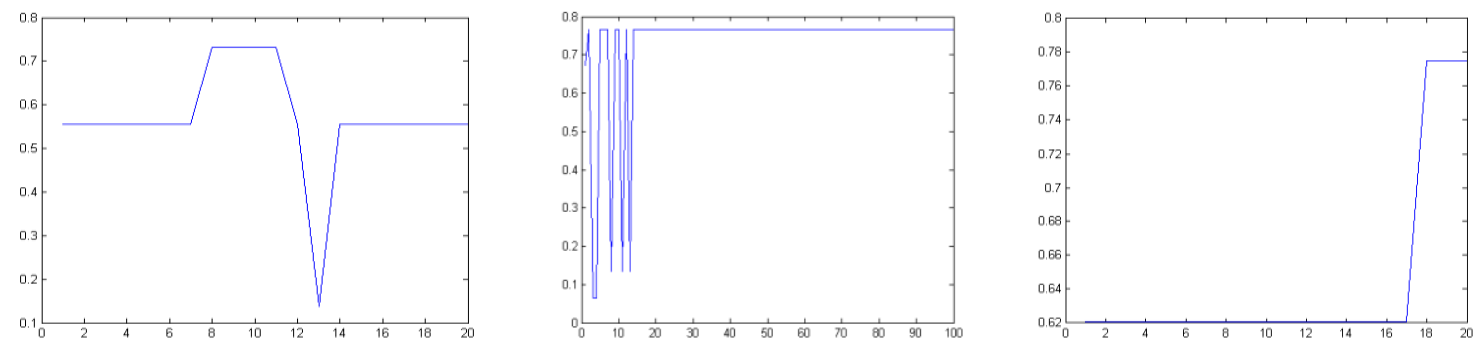

Fig. 3. Results for cost a) MOIA b) MOMBO c) MOSA (from left to right).

Of course, we have to simultaneously consider both availability and cost. We think the result of MOMBO is more suitable than those of the other algorithms. Since algorithms proceed randomly, running by others may produce different results, but in the expanded viewpoint, the results produced are the same as the results presented.

\section{CONCLUSION}

One of the major services in LTE is video streaming. The quality of the video stream is important from the 
viewpoint of the users. In this paper, we present QoE in LTE as an optimization problem. Hence, it becomes easily comprehensible for users and the administrator. The QoE presented has multiple objective functions, so we use multi-objective optimization algorithms. Three algorithms are used in our work: 1- Multi-Objective Monarch Butterfly Optimization (MBO), 2- MultiObjective Intelligent Automata (MOIA) and 3- MultiObjective Simulated Annealing (MOSA). There is no need for a comparison between individuals in $\mathrm{MBO}$, but in other algorithms, comparisons between individuals are required. Thus, the multi-objective release of $\mathrm{MBO}$ is the same as a single-objective $\mathrm{MBO}$, but we present a new release of MOSA and MOIA.

\section{REFERENCES}

[1] S. Mavoungou, G. Kaddoum, M. Taha, and G.Matar, "Survey on threats and attacks on mobile networks," IEEE, Special Section On Security in Wireless Communications and Networking, 2016.

[2] V. Bagubali, P. Prithiviraj, and S. Mallick, "Performance analysis of IMS based LTE and WIMAX integration architectures," Alexandria Engineering Journal, 2016.

[3] S. AlQahtani, "Users' classificationbased call admission control with adaptive resource reservation for LTE-A Networks," Journal of King Saud University - Computer and Information Sciences, 2016.

[4] S. Mathi and L. Dharuman, "Prevention of desynchronization attack in 4G LTE networks using double authentication scheme," Procedia Computer Science, vol. 89, pp. 170-179, 2006.

[5] T. Padmapriya and V. Saminadan, "Handoff decision for multiuser multi-class traffic in MIMOLTE-A networks," Procedia Computer Science, vol. 92, pp. 410-417, 2006.

[6] T. Ghalut, H. Larijania, and A. Shahrabia, "QoE-aware optimization of video stream downlink scheduling over LTE networks using RNNs and genetic algorithm," Procedia Computer Science, vol. 94, 232-239, 2006.

[7] A. Gago and N. Cota, "Performance evaluation of femtocells usage on LTE," Procedia Technology, vol. 17, pp. 683-691, 2007.

[8] M. Abdeljebbar and R. El Kouch, "Fast authentication during handover in 4G LTE/SAE networks," IERI Procedia 10, pp. 11-18, 2014.

[9] L. Deng, Z. Chen, and Y. Zhao, "Basis expansion model for channel estimation in LTE-R communication system," Digital Communications and Networks, pp. 92-96, 2016.

[10] W. Kim, G. Seok Park, and H. Song, "An effective cross-layer designed packet scheduling, call admission control, and handover system for video streaming services over LTE network," J. Vis. Commun. Image R. vol. 31, 2015.

[11] A. Carlos, A. David, V. Veldhuizen, and B. Lamont, "Evolutionary algorithms for solving multi-objective problems," Speringer, p. 74, 2007.

[12] C. Dimopoulos, "A review of evolutionary multi-objective optimization, application in the area of production research," IEEE, vol. 2, 2004.

[13] G. Wang, S. Deb, X. Zhao, and Z. Cui, "A new monarch butterfly optimization with an improved crossover operator," Springer, 2016.

[14] G. Wang, X. Zhao, and S. Deb, "A novel monarch butterfly optimization with greedy strategy and self-adaptive crossover operator," in Proc. Second International Conference on Soft Computing and Machine Intelligence, 2015.

[15] G. Wang, S. Deb4, and Z. Cui, "Monarch butterfly optimization," Theory and Applications of Soft Computing Methods, 2015.

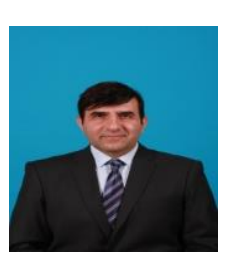

Seyed Mahmood Hashemi received his bachelor from Islamic Azad University (Qazvin Branch) in software engineering at 2001, his master from Islamic Azad University (Science and Research Branch) in arti_cial intelligence at 2003. He is currently Ph.D candidate in Beijing University of Technology (BJUT). His research interests are internet of things (IoT), network security and artificial intelligence (AI). 